Moreover, the tumorigenicity was abolished by PMN-MDSC depletion. Notably, intrasplenic injection of a mouse melanoma cell line B16F10 exhibited an increased level of liver-infiltrating PMN-MDSCs and enhanced liver metastasis in male TG mice compared to control mice. Moreover, depletion of PMN-MDSCs suppressed metastasis in liver. Mechanistically, anti-tumor NKT cells, rather than NK cells and $\mathrm{CD} 8^{+} \mathrm{T}$ cells, were negatively correlated with tumor weight and MDSC proportion, indicating involvement of cross-talk between MDSC and NKT in liver metastasis.

Conclusions Our findings suggest that hepatic CCRK expression create a tumor growth- and metastasis-supportive liver microenvironment via enhancing immunosuppression.

\section{IDDF2019-ABS-0266 EFFECTS OF THYROID HORMONE TREATMENT ON HEPATIC GLUCOSE PRODUCTION AND RENAL REABSORPTION OF GLUCOSE IN ALLOXAN-INDUCED DIABETIC WISTAR RATS}

Ranbir Singh*. Department of Applied Sciences, Raja Mahendra Pratap Post Graduate College, Haridwar (Uttarakhand), India

\subsection{6/gutjnl-2019-IDDFabstracts. 106}

Background The thyroid hormone (TH) plays an important role in glucose metabolism. Recently, we showed that the TH improves glycemia control by decreasing cytokines expression in the adipose tissue and skeletal muscle of alloxan-induced diabetic rats, which were also shown to present primary hypothyroidism. In this context, this study aims to investigate whether the chronic treatment of diabetic rats with T3 could affect other tissues that are involved in the control of glucose homeostasis, as the liver and kidney.

Methods Adult male Wistar rats were divided into nondiabetic, diabetic, and diabetic treated with T3 $(1.5 \mu \mathrm{g} / 100 \mathrm{~g}$ BW for 4 weeks). Diabetes was induced by alloxan monohydrate $(150 \mathrm{mg} / \mathrm{kg}, \mathrm{BW}$, i.p.). Animals showing fasting blood glucose levels greater than $250 \mathrm{mg} / \mathrm{dL}$ were selected for the study.

Results After treatment, we measured the blood glucose, serum T3, T4, TSH, and insulin concentration, hepatic glucose production by liver perfusion, liver PEPCK, GAPDH, and pAKT expression, as well as urine glucose concentration and renal expression of SGLT2 and GLUT2. T3 reduced blood glucose, hepatic glucose production, liver PEPCK, GAPDH, and pAKT content and the renal expression of SGLT2 and increased glycosuria.

Conclusions Results suggest that the decreased hepatic glucose output and increased glucose excretion induced by T3 treatment are important mechanisms that contribute to reducing serum concentration of glucose, accounting for the improvement of glucose homeostasis control in diabetic rats.

\section{IDDF2019-ABS-0267 EPIDEMIOLOGICAL STUDY OF THE PREVALENCE OF HEPATITIS B VIRUS IN SCHOOL-AGE CHILDREN IN WEST INDIAN STATE}

Tanvi Sharma*, Vijay Sharma. Regional Institute of Health Medicine and Research, Jaipur, Rajasthan, India

10.1136/gutjnl-2019-IDDFabstracts. 107
Background In India, chronic hepatitis B is acquired predominantly by horizontal transmission in early childhood and to a lesser extent by perinatal transmission. The exact mode of horizontal transmission remains undefined, may be close contact, body fluid contact. The age of acquisition of HBV is an important determinant of outcome; (e.g., $>90 \%$ in newborns (vertical transmission), 30\% in children aged 2-5 years and $<5 \%$ in adults). HBV also spread by parenteral transmission at any age including intravenous drug use, unsafe therapeutic injections, occupational injuries or nosocomial transmission. There is no data of HBV prevalence among school children in the western Indian state of Rajasthan so we decided to conduct an epidemiological study in the year 2018-2019.

Methods Senior liver expert designed and planned the study, school administration, social workers and school student volunteers were involved in arranging HBV test camps at government schools. Discussion, presentations, study material distribution, coordination, data collection was done by students under the supervision of team, five government school in Jaipur district were covered, HBV ELISA spot test was done.

Results Total 1255 students were screened, out of them 17 were positive but asymptomatic, 11 male, 6 female, age 10 to 16 years. The study suggests a lower prevalence of $\mathrm{HBV}$ in west India likely due to high vaccination coverage. Old studies suggest rates of HBsAg-positivity 2.14-2.25\% among children $<5$ years of age and $4.3-7.2 \%$ among the entire paediatric population, while $6.7 \%$ among those with liver disease, $2 / 3 \mathrm{rd}$ $\mathrm{HBeAg}$ negative. Though horizontal transmission is the predominant mode, the contribution of vertical transmission is also important. Hepatitis B is a significant public health problem in India, yet disease awareness among Indian people, the key to decreasing disease burden is dismally low. The majority of disease cases progress silently and patients present in advanced stages when decompensated CLD or HCC has already developed.

Conclusions Prevalence of $\mathrm{HBV}$ is $1.35 \%$ in west Indian school kids, countrywide vaccination coverage should be more aggressive in the pediatric population. Our emphasis should be on health education of general and high-risk populations along with aggressive vaccination strategies especially for tribals and high-risk groups.

\section{IDDF2019-ABS-0302 IDENTIFICATION OF DNA METHYLATION SIGNATURES FOR MICROVASCULAR INVASION IN HEPATOCELLULAR CARCINOMA}

Lixia Xu*, Sui Pen, Qiye He, Zhihang Chen, Ming Kuang. The First Affiliated Hospital, Sun Yat-sen University, China

\subsection{6/gutjnl-2019-IDDFabstracts. 108}

Background The presence of microvascular invasion (MVI) reduces overall survival of hepatocellular carcinoma (HCC). Recent studies showed DNA methylation markers could be applied to the diagnosis of cancers. However, it is unclear whether DNA methylation signatures could help diagnose $\underline{\mathrm{MVI}}$ in HCC.

Methods To identify DNA methylation markers for HCC and MVI diagnosis, we first generated genome-wide DNA methylation profiles from HCC tissues and adjacent normal liver 